This item was submitted to Loughborough's Research Repository by the author.

Items in Figshare are protected by copyright, with all rights reserved, unless otherwise indicated.

\title{
The SAR effects of popular jewellery on the human head
}

PLEASE CITE THE PUBLISHED VERSION

LICENCE

CC BY-NC-ND 4.0

\section{REPOSITORY RECORD}

Whittow, W.G., C.J. Panagamuwa, R.M. Edwards, and J.C. Vardaxoglou. 2019. "The SAR Effects of Popular Jewellery on the Human Head". figshare. https://hdl.handle.net/2134/3057. 
This item was submitted to Loughborough's Institutional Repository by the author and is made available under the following Creative Commons Licence conditions.

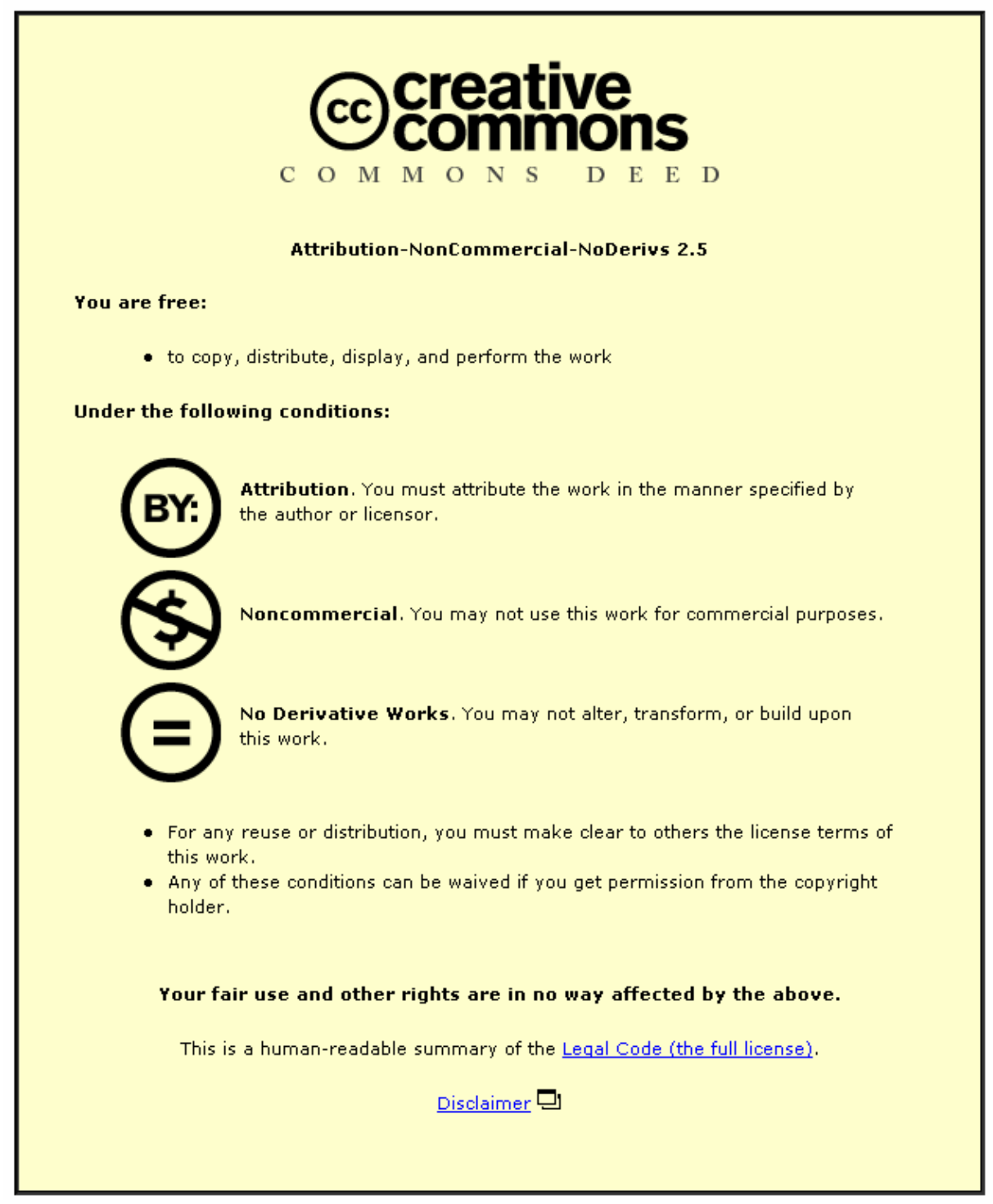

For the full text of this licence, please go to: http://creativecommons.org/licenses/by-nc-nd/2.5/ 


\title{
THE SAR EFFECTS OF POPULAR JEWELLERY ON THE HUMAN HEAD
}

\author{
W. G. Whittow ${ }^{\dagger}$, C. J. Panagamuwa ${ }^{\dagger}$, R. M. Edwards ${ }^{\dagger}$, J. C. Vardaxoglou ${ }^{\dagger}$ \\ ${ }^{\dagger}$ Electronic and Electrical Engineering Dept., Loughborough University, Leicestershire, LE11 3TU. UK, \\ w.g.whittow@lboro.ac.uk,c.j.panagamuwa@lboro.ac.uk,r.m.edwards@lboro.ac.uk, \\ j.c.vardaxoglou@lboro.ac.uk
}

Keywords: SAR, FDTD, DASY, Metallic jewellery

\begin{abstract}
This paper investigates the effects of metallic jewellery on the $\mathrm{SAR}$ in the human head. A CW dipole is placed in front of the head to represent a mobile enabled personal data assistant. The FDTD method has been used to simulate an eyebrow ring near a homogeneous SAM phantom at $1.8 \mathrm{GHz}$. Measurements were made on the Loughborough SAM head with the DASY4 measurement system. Simulations were also made with eyebrow rings on the surface of the skin and pierced through the eyebrow of a heterogeneous anatomically realistic human head. Common sizes of eyebrow ring and eyebrow stud have been considered over the frequency range 0.6 to $4.6 \mathrm{GHz}$. Jewellery which was small compared to a wavelength had little effect on the SAR in the head.
\end{abstract}

\section{Introduction}

There is public concern and scientific interest in the possibility that the radiation from mobile phones may adversely affect human health. The international standard to quantify these effects is Specific Absorption Rate (SAR). In previous works, the authors [1] [2] have found that metallic spectacles can increase the SAR in the eyes and head. This paper investigates the effects of metallic jewellery on the SAR in the human head. It is commonly estimated that there are a billion mobile phone users in the world. Many of these may also wear metallic jewellery or have metallic objects in close proximity to their head. These include facial piercings, metallic spectacles, jewellery on the hands, hairclips, and Bluetooth microphones. This paper will consider eyebrow rings and eyebrow studs.

In the Finite-Difference Time-Domain (FDTD) simulations, we have used a continuous wave (CW) source, positioned in front of the head to study the effects of a communications enabled personal data assistant mobile communications equipment (PDAMCE) device, used in front but away from the face. In recent years, work has been presented regarding mobile communications equipment (MCE) positioned near the ear [3-6]. The head has also been irradiated from in front of the eye using realistic mobile phone models [5-7].
Recently, the authors have briefly considered the effect of metallic jewellery on the SAR in the head [8]. Virtanen [9] found that metallic implants inside the head could increase the SAR. She considered metallic loops and pins inside a cylindrical head. The same author has reviewed the area of passive implants [10] and investigated implants in a heterogeneous anatomical model of a head [11]. Tay [12] has investigated the change in radiation efficiency using a passive reflector and directive element. He found that the efficiency of the reflector could be increased if a metallic scatterer was positioned near to a dipole.

In the same area Cooper [13], modelled a geometric head, and Bernardi [14] investigated an anatomical head, irradiated by simple dipoles positioned near conducting surfaces. Both found that such systems could increase the power absorbed in the head. In [15] Cooper considered metal implants inside the head and found that they increased the SAR in the surrounding region. These papers show that metal objects close to biological matter may increase SAR in that matter. Troulis [16] used the FDTD method to briefly examine thin metallic spectacles on a heterogeneous phantom with a resolution of $5 \mathrm{~mm}$. The excitation used was a monopole on a metallic box positioned at the side of the head. The paper showed that metallic spectacles may re-distribute energy produced by MCE. A drop in the efficiency of the MCE antenna was associated with SAR increase.

Griffin [17] performed measurements with a phantom and metallic spectacles and showed that spectacles can increase or decrease the level of radiation near the eyes by up to $20 \mathrm{~dB}$ due to shielding, enhancement and depolarization effects. Anderson [18] also performed measurements with a phantom wearing spectacles. For MCE operating at $835 \mathrm{MHz}$, held by the ear, the SAR in the eye closest to the phone was found to increase by almost 30\%. Wang [19] modelled a monopole on a metallic box positioned by the ear of a human head wearing spectacles at $1.5 \mathrm{GHz}$ using the FDTD method. The average SAR in the eye increased by up to 2.7 times. Wang postulated that increased SAR in parts of the head was due to current on the spectacles. Joo [20] used the FDTD method and scaled models of an adult head to consider the effects of metallic spectacles on adults and children with a mobile phone placed by the ear. In the same paper, he also considered passive implants by the ear. Metallic ear rings have previously been considered with a source by the ear [11] [21] [22]. 


\section{Description of Model}

An independent 3D FDTD code [1] [2] has been written; see Taflove [23] for an excellent reference. Perfectly Matched Layers (PML), with geometric grading [24], absorbing boundary conditions are used to terminate the grid. The PML is eight cells thick and is positioned at least twelve cells from the head. The Yee cell size used throughout this paper is $2 \mathrm{~mm}$. The lowest number of cells per wavelength was always greater than ten at $1.8 \mathrm{GHz}$, and reasonable results have been obtained with only four [3] [25]. At 4.6GHz, the number of cells per wavelength is greater than 4.5. The time step was $3.336 \mathrm{pS}$. The simulations were run for at least ten cycles and until stability was achieved (at least 1660 time steps at $1.8 \mathrm{GHz}$ ). The geometry of the problem space is as follows; the $X$ axis runs from the tip of the nose to the back of the head, the $\mathrm{Y}$ axis is from ear to ear and the $\mathrm{Z}$ axis runs from chin to top of the head.

SAR is the standard criteria to measure the amount of electromagnetic energy absorbed in the body and is calculated as in equation (1)

$$
S A R=\frac{\sigma|E|^{2}}{\rho} \quad(\mathrm{W} / \mathrm{kg})
$$

Where $|E|$ is the rms magnitude of the electric field strength vector, $\rho$ is the mass density of the material in $\mathrm{kg} / \mathrm{m}^{3}$ and $\sigma$ is the electrical conductivity in $\mathrm{S} / \mathrm{m}$. The SAR is calculated with the twelve-field approach as used by Caputa [26].

\subsection{Dipole model}

To allow comparison between simulations and measurements, a dipole model [23] has been used as the excitation in this paper. The dipole is vertically orientated along the $\mathrm{Z}$ axis (from chin to top of head) and fed at its centre with a sinusoidal CW source. In the FDTD simulation the dipole was modelled by setting the tangential E-field components to zero along the length of the dipole.

\subsection{The head model}

Section 3.1 of this paper uses the Loughborough SAM head. The rear of the head has been removed to allow the head to be placed down so measurements can be made in the face of the SAM head [27] [28]. The properties of the brain simulating tissue of the homogeneous head are $(\sigma=1.37 \mathrm{~S} / \mathrm{m}, \varepsilon \mathrm{r}=40.48$, $\left.\rho=1000 \mathrm{~kg} / \mathrm{m}^{3}\right)$. The properties of the shell are $(\sigma=0 \mathrm{~S} / \mathrm{m}$, $\varepsilon r=3.5)$.

In sections 3.2 and 3.3 of the results, metallic jewellery is added to an anatomically realistic head. A head matrix provided by Brooks Air force (www.brooks.af.mil) was used. The head, which is based on The Visible Human Project, is that of an adult male and has twenty-five tissue types. The head data has a $2 \mathrm{~mm}$ resolution.

\subsection{Modelling the metallic jewellery}

The metallic jewellery was modelled using metallic Yee cells, by setting the conductivity of the cells equal to the conductivity of copper [1]. Nikita [29] and Bernardi [14] both used this technique to model metal shapes. Wang [19] modelled metallic spectacles by setting the conductivity of the Yee cells in the frames equal to titanium. In the range of conductivities used amongst these researchers and ourselves, changes to SAR given by equation (1) are not significant. The rings and studs investigated in this paper were $2 \mathrm{~mm}$ thick (1 Yee cell). The studs were orientated along the $\mathrm{Z}$ axis and therefore parallel to the dipole. Studs orientated perpendicular to the dipole was found to have negligible effect.

\section{Results}

\subsection{Eyebrow ring touching $\mathrm{SAM}$ head at $1.8 \mathrm{GHz}$}

In this section, an eyebrow ring has been added to the eyebrow of the Loughborough SAM head at $1.8 \mathrm{GHz}$. The simulation setup is similar to the measurement setup shown in Figure 1. The dipole was positioned $80 \mathrm{~mm}$ (along the $\mathrm{X}$ axis) in front of the tip of the nose and orientated in the $Z$ direction. The centre of the dipole in the $Z$ axis was level with the eyebrows. The dipole and the edge of the ring were located $32 \mathrm{~mm}$ to the side of the head (along the $\mathrm{Y}$ axis) to lie at the at the centre of the eyebrow. The edge of the ring touched the eyebrow in the same position as the ring size was varied.



Figure $1.52 \mathrm{~mm}$ eyebrow ring touching SAM head.

The maximum $1 \mathrm{~g}$ and $10 \mathrm{~g}$ SAR with various sizes of eyebrow ring found with the FDTD code are shown in Figure 2. The rings have negligible effect if the circumference of the ring is less than $0.7 \lambda$. As the size of the ring was increased beyond this size the $1 \mathrm{~g}$ and $10 \mathrm{~g}$ SAR increased. The maximum diameter of ring considered was $52 \mathrm{~mm}$ (circumference $=098 \lambda$ ). This size meant the eyebrow ring did not extend 
beyond the edge of the head. Figure 2 shows the maximum SAR occurs when the circumference of the eyebrow ring is approximately one wavelength. The $52 \mathrm{~mm}$ diameter ring increases the $1 \mathrm{~g} \mathrm{SAR}$ by 3.8 times from 1.36 to $5.12 \mathrm{~W} / \mathrm{kg}$. The $10 \mathrm{~g}$ SAR is increased by 3.0 times from 0.62 to $1.83 \mathrm{~W} / \mathrm{kg}$.

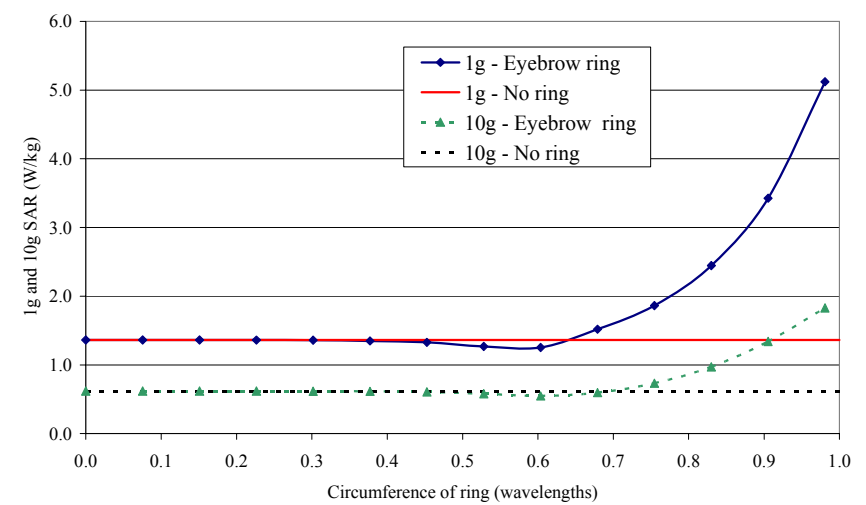

Figure 2. FDTD simulations of different sizes of eyebrow ring near the SAM head.

The simulated results in Figure 2 with and without the $52 \mathrm{~mm}$ eyebrow ring were repeated with the Loughborough SAM head measurement system [27] [28]. The measured and simulated results are compared in Table 1.

\begin{tabular}{|c|c|c|c|c|}
\hline & $\begin{array}{c}\text { FDTD - } \\
\text { No ring }\end{array}$ & $\begin{array}{c}\text { DASY- } \\
\text { No ring }\end{array}$ & $\begin{array}{c}\text { FDTD - } \\
52 \mathrm{~mm} \text { ring }\end{array}$ & $\begin{array}{c}\text { DASY - } \\
52 \mathrm{~mm} \text { ring }\end{array}$ \\
\hline $1 \mathrm{~g}$ & 1.36 & 1.95 & 5.12 & 3.99 \\
\hline $10 \mathrm{~g}$ & 0.62 & 0.71 & 1.83 & 1.84 \\
\hline
\end{tabular}

Table $1.1 \mathrm{~g}$ and $10 \mathrm{~g} \mathrm{SAR}(\mathrm{W} / \mathrm{kg}$ ) for DASY measurements and FDTD simulations with and without $52 \mathrm{~mm}$ eyebrow ring.

The results show that the eyebrow ring significantly increased the SAR in the head. The $10 \mathrm{~g}$ SAR results show good agreement with the FDTD simulations. The $1 \mathrm{~g}$ SAR measurement results are increased with the ring but the relative increase due to the ring is smaller than with the FDTD results. The $1 \mathrm{~g}$ averaging volume is much smaller than the $10 \mathrm{~g}$ volume and the $1 \mathrm{~g}$ results are consequently much more sensitive. Although, the $1 \mathrm{~g}$ SAR results for FDTD simulations and DASY measurements are not the same they are on the same order of magnitude and both sets show an increase in SAR. We believe that slight differences in position of the ring in the simulations compared to the measurements combined with staircasing effects in the FDTD model contribute to the differences in results. The maximum SAR without the ring lies behind the nose at the centre of the head. With the ring, the maximum SAR occurs at the centre of the eyebrow, where the ring touches the head. Therefore, the maximum SAR, without the pin, in the DASY4 measurements lies behind the seam that joins the two half heads together, see Figure 1. This seam may act as a lossless adaptor that improves the match between the air and the liquid and increases the SAR. Bernardi [30] found a similar phenomem with a glass lens of a pair of spectacles. Note, previously the authors found excellent agreement between simulations and measurements with a ring near a flat phantom. Good agreement has also been found with a straight metallic jewellery pin in front of the Loughborough SAM head [31].

\subsection{Eyebrow ring near heterogeneous head at $1.8 \mathrm{GHz}$}

In this section, the effect of a metallic eyebrow ring near the heterogeneous Brooks head is investigated. As in section 3.1, the dipole was positioned $80 \mathrm{~mm}$ in front of the nose and in front of the eyebrow and at a height level with the centre of the ring. The diameter of the eyebrow ring was varied from 0 to $60 \mathrm{~mm}$. Four locations of ring were considered; pierced through the skin (replacing the tissues with copper Yee cells), touching the surface of the skin, $4 \mathrm{~mm}$ in front of the eyebrow and $8 \mathrm{~mm}$ in front of the eyebrow. Rings positioned away from the head could include certain design of spectacles, metallic jewellery coated in a lossless plastic or future fashions in piercings and jewellery.

The $1 \mathrm{~g}$ SAR with the eyebrow ring is shown in Figure 3. Rings with a circumference of less than $0.6 \lambda$ have negligible effect. Larger rings can increase the $1 \mathrm{~g}$ SAR by up to 3.5 times. When the ring is touching the skin surface at the eyebrow the maximum effect occurs when the circumference is approximately one wavelength. When the ring is pierced through the skin, the ring resonates at a larger size. Therefore, a ring of a specific size (and larger than $0.6 \lambda$ ) could have different effects depending whether it was pierced through the skin or on the skin's surface. If the eyebrow ring was positioned 4 or $8 \mathrm{~mm}$ in front of the eyebrow, all sizes of ring had negligible effect.

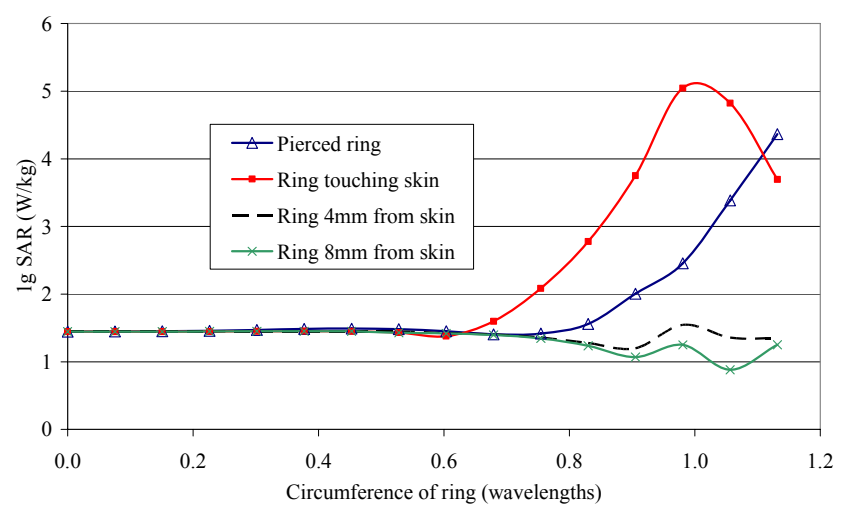

Figure 3. 1g SAR when varying the position and size of eyebrow ring near the heterogeneous Brooks head.

Figure 4 shows the $10 \mathrm{~g} \mathrm{SAR}$, as the position and size of an eyebrow ring is varied, with the heterogeneous Brooks head. The rings have little effect until the circumference is larger than half a wavelength. The figure shows that eyebrow rings of different sizes and positions do not increase the $10 \mathrm{~g}$ SAR 
by significant amounts. Large rings further from the face can decrease the $10 \mathrm{~g}$ SAR.

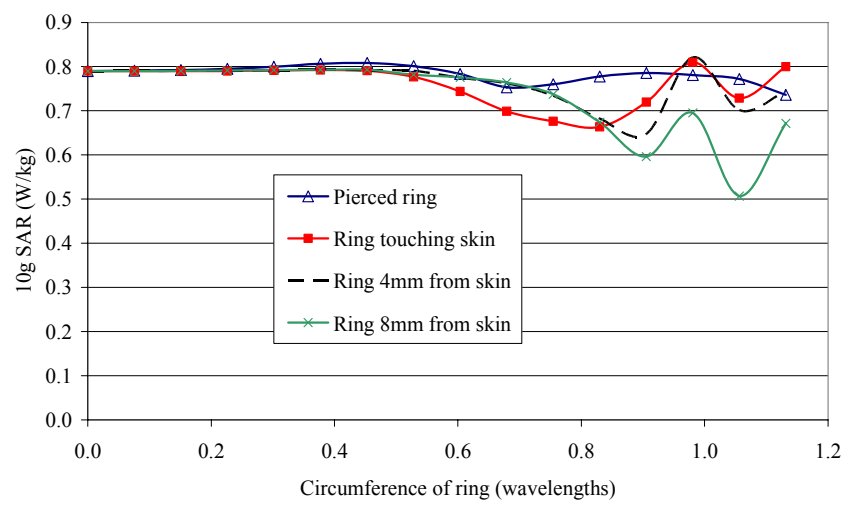

Figure 4. $10 \mathrm{~g}$ SAR when varying the position and size of eyebrow ring near the heterogeneous Brooks head.

Previously, the authors [8] have found that the distance of metallic jewellery from a cubic phantom was very significant. Negligible effect was found with the jewellery close to the cubic head. The maximum effect was with the jewellery $12 \mathrm{~mm}$ from the cube and the SAR could be reduced when the jewellery was moved further away from the cubic head [8]. Clearly, the geometry with a realistically shaped head is much more complicated than the cube, as different parts of the ring are different distances from the head. Therefore, the difference between the pierced ring and the ring on the surface could be partially due to the fact that the pierced ring is $4 \mathrm{~mm}$ further away from the dipole and therefore $4 \mathrm{~mm}$ closer to parts of the face. If the ring is touching the eyebrow, the curvature of the head means that most of the ring is a couple of centimetres away from the face. This distance is further increased if the ring is then moved 4 or $8 \mathrm{~mm}$ away from the eyebrow.

\subsection{Popular jewellery on an anatomical head over a range of frequencies}

This section investigates the effects of popular sizes of jewellery on an anatomical head over a range of frequencies that include the communication spectra. The types of jewellery considered are; a $12 \mathrm{~mm}$ diameter eyebrow ring and a $10 \mathrm{~mm}$ long eyebrow stud. These types of jewellery are currently very common. The eyebrow stud is orientated parallel to the dipole. For all the FDTD results in this section, the dipole is positioned central to the head. The frequency range is 0.6 to $4.6 \mathrm{GHz}$. The jewellery has been considered to both lie on the skin surface and to be pierced through the skin.

Figure 5 shows the $1 \mathrm{~g}$ SAR results with each piece of jewellery. The results show that these sizes of metallic jewellery have little effect at communication spectra. Note, the jewellery is relatively small compared to a wavelength and the $1 \mathrm{~g}$ SAR with jewellery is very similar to the $1 \mathrm{~g} \mathrm{SAR}$ without jewellery. At lower frequencies in this range, which have larger wavelengths, the jewellery considered has negligible effect. Above $3 \mathrm{GHz}$, jewellery begins to have a small effect. At frequencies below $4.6 \mathrm{GHz}$, small popular jewellery (both rings and studs) have little effect on the SAR. This is true whether it is pierced through the skin or lies on the skin surface. The $1 \mathrm{~g}$ SAR is increased or decreased by less than $2.5 \%$ at frequencies up to $3 \mathrm{GHz}$. The $1 \mathrm{~g}$ SAR variation increases to $\pm 10 \%$ when the frequency range is extended to $4.6 \mathrm{GHz}$.

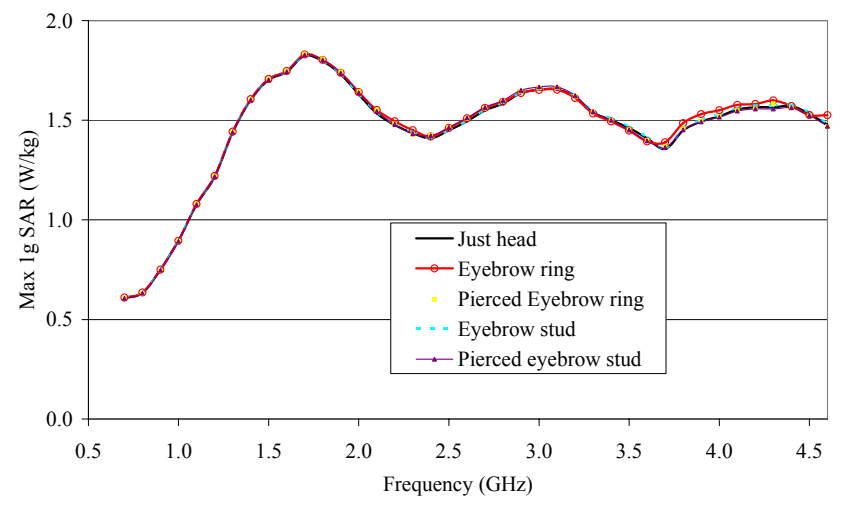

Figure 5. $1 \mathrm{~g}$ SAR with common jewellery on the face of the anatomical head over a frequency range.

Figure 6 shows the $10 \mathrm{~g}$ SAR results with the same pieces of jewellery (both pierced through the skin and on the skin's surface) over the frequency range 0.6 to $4.6 \mathrm{GHz}$. The results are similar to the $1 \mathrm{~g}$ SAR results shown in Figure 5. The addition of jewellery affects the $10 \mathrm{~g}$ SAR by less than $2 \%$ over the frequency range considered.

Both the eyebrow ring and stud, either pierced through the skin or on the skin's surface, increased or decreased the average SAR in the eye closest to the ring by less than $7 \%$ over the frequency range considered. The jewellery also had negligible effect on the average SAR in the head. The change in the average SAR in the head was less than $1 \%$ up to $4.3 \mathrm{GHz}$. The eyebrow ring on the surface of the head increased the average SAR in the head by $4 \%$ at $4.6 \mathrm{GHz}$.



Figure 6. 10g SAR with common jewellery on the face of the anatomical head over a frequency range. 


\section{Conclusions}

This paper has investigated the effects of popular jewellery at communication frequencies with the excitation in front of the face. At $1.8 \mathrm{GHz}$, a metallic ring touching the fibreglass shell of the homogeneous SAM phantom, increased the 1g SAR by 4 times when the ring was approximately one wavelength in circumference. The $10 \mathrm{~g}$ SAR increased by three times.

The eyebrow ring had similar effects on the $1 \mathrm{~g}$ SAR with the heterogeneous anatomical head. Differences were observed between an eyebrow ring pierced through the skin and touching the surface of the skin. Larger sizes of rings resonated when the rings were pierced through the skin. Note, only a small section of the ring was pierced through the skin and most of the ring was not touching the skin due to the curvature of the head. The eyebrow rings marginally decreased the $10 \mathrm{~g}$ SAR in the heterogeneous head. Rings positioned several millimetres from the head did not increase the SAR in the head. Note, that at communication frequencies, wavelengths are likely to be larger than common jewellery sizes.

Popular sizes of eyebrow and eyebrow studs on an anatomical head have also been examined over the frequency range 0.6 to 4.6GHz. The addition of this jewellery had negligible effect on the $1 \mathrm{~g}, 10 \mathrm{~g}$ SAR or the average SAR in the eye over the spectra considered. This observation was true when the jewellery was pierced through the skin or resting on the skin's surface.

Metallic jewellery can significantly increase the SAR in the head if its size is of the order of a wavelength. If the jewellery is much smaller than this, it is unlikely to have a significant effect. Therefore, at communications spectra, current facial jewellery such as nose ring, eyebrow rings or small piercings are not expected to have a large effect. However, other objects such as ear rings or metallic spectacles, which are generally closer to a wavelength in size, may increase or decrease the SAR. It is also beyond the scope of this paper to predict future trends of fashion and what might become popular in years to come.

The results presented here with an eyebrow ring positioned away from the head differ from those with spectacles [1]. This shows that similar sized metallic objects behave differently on different parts of the head. Therefore, jewellery on a heterogeneous realistically shaped head is much more complicated to analyse that jewellery near a cubic head and each size and shape of jewellery needs to be investigated separately on different parts of the head. The situation is further complicated as the effect of piercing the object through the skin alters the SAR. The effect of the jewellery is also likely to vary with different heads models. Therefore, an alternative approach to investigating all the combinations of jewellery that exist currently and could become popular in the future, is to simulate and measure jewellery on cubic heads. This allows the general enhancement mechanisms to be understood and the order of magnitude of the relative effect of the jewellery can be investigated. There is an infinite number of combinations of sizes and positions of jewellery, so a simple cubic model could be used to provide a worse case scenario in terms of maximum SAR.

\section{References}

[1] W. G. Whittow and R. M. Edwards, "A study of changes to specific absorption rates in the human eye close to perfectly conducting spectacles within the radio frequency range 1.5 to $3.0 \mathrm{GHz}$.," IEEE Trans. Antennas and Propagation, vol. 52, pp. 3207-3212, 2004.

[2] W. G. Whittow and R. M. Edwards, "Applications of a genetic algorithm for identification of maxima in specific absorption rates in the human eye close to perfectly conducting spectacles," IEE Proceedings Science, Measurement \& Technology, vol. 152, pp. 89-96, 2005.

[3] A. D. Tinniswood, C. M. Furse, and O. P. Gandhi, "Computations of SAR distributions for two anatomically based models of the human head using CAD files of commercial telephones and the parallelized FDTD code," IEEE Transactions on Antennas and Propagation, vol. 46, pp. 829-833, 1998.

[4] O. Gandhi, G. Lazzi, and C. M. Furse, "Electromagnetic absorption in the human head and neck for mobile telephones at 835 and 1900MHz," IEEE Trans. Microwave Theory Technology, vol. 44, pp. 1884-1897, 1996.

[5] P. J. Dimbylow and S. M. Mann, "SAR calculations in an anatomically realistic model of the head for mobile communication transceivers at $900-\mathrm{MHz}$ and 1.8-GHz," Physics in Medicine and Biology, vol. 39, pp. 1537-1553, 1994.

[6] P. Wainwright, "Thermal effects of radiation from cellular telephones," Physics in Medicine and Biology, vol. 45, pp. 2363-2372, 2000.

[7] L. Martens, J. Demoerloose, D. Dezutter, J. Depoorter, and C. Dewagter, "Calculation of the electromagnetic-fields induced in the head of an operator of a cordless telephone," Radio Science, vol. 30 , pp. 283-290, 1995.

[8] W. Whittow, C. J. Panagamuwa, R. Edwards, J. C. Vardaxoglou, and P. McEvoy, "A study of head worn jewelry, mobile phone RF energy and the effect of differing tissue types on rates of absorption," in The First European Conference on Antennas and Propagation (EuCAP 2006), Nice, France, 2006.

[9] H. Virtanen, J. Huttunen, A. Toropainen, and R. Lappalainen, "Interaction of mobile phones with superficial passive implants," Physics in Medicine and Biology, vol. 50, pp. 2689-2700, 2005.

[10] H. Virtanen, J. Keshvari, and R. Lappalainen, "Interaction of Radio Frequency electromagnetic 
fields and passive metallic implants-a brief review," Bioelectromagnetics, vol. 27, pp. 431-439, 2006.

[11] H. Virtanen, J. Keshvari, and R. Lappalainen, "The effect of authentic metallic implants on the SAR distributions of the head exposed to 900, 1800 and 2450MHz dipole near field," Physics in Medicine and Biology, vol. 52, pp. 1221-1226, 2007.

[12] R. Tay, Q. Balzano, and N. Kuster, "Dipole configurations with strongly improved radiation efficiency for hand-held transceivers," IEEE Trans. Antennas and Propagation, vol. 46, pp. 798-806, 1998.

[13] J. Cooper and V. Hombach, "The specific absorption rate in a spherical head model from a dipole with metallic walls nearby," IEEE Transactions on Electromagnetic Compatibility, vol. 40, pp. 377-382, 1998.

[14] P. Bernardi, M. Cavagnaro, and S. Pisa, "Evaluation of the SAR distribution in the human head for cellular phones used in a partially closed environment," IEEE Transactions on Electromagnetic Compatibility, vol. 38, pp. 357-366, 1996.

[15] J. Cooper and V. Hombach, "Increase in specific absorption rate in humans from implantations," Electronic Letters, vol. 32, pp. 2217-2219, 1996.

[16] S. E. Troulis, W. G. Scanlon, and N. E. Evans, "Effect of 'hands-free' leads and spectacles on SAR for a $1.8 \mathrm{GHz}$ cellular handset.," in 1st Joint IEI / IEE Symposium on Telecommunications Systems Research, Dublin, 2001, pp. 1675-1684.

[17] D. W. Griffin, "A microwave antenna method of measuring the effect of metal-framed spectacles on microwaves near the eye.," Antennas and Propagation Society International Symposium, vol. 21, pp. 253-256, 1983.

[18] V. Anderson and K. H. Joyner, "Specific absorption rate levels measured in a phantom head exposed to radiofrequency transmissions from analog hand-held mobile phones," Bioelectromagnetics, vol. 16, pp. 60-69, 1995.

[19] J. Wang, T. Joukou, and O. Fujiwara, "Localized specific absorption rate in the human head in metalframed spectacles for $1.5 \mathrm{GHz}$ Hand-held mobile telephones," Trans IEE of Japan, vol. 118-A, pp. 1234-1240, 1998.

[20] E. Joo, A. Szasz, and P. Szendro, "Metal-framed spectacles and implants and specific absorption rate among adults and children using mobile phones at 900/1800/2100MHz," Electromagnetic Biology and Medicine, vol. 25, pp. 103-112, 2006.

[21] W. Whittow, C. Panagamuwa, R. Edwards, and J. Vardaxoglou, "Specific absorption rates in the human head due to circular metallic earrings at 1800MHz," in 2007 Loughborough Antennas and Propagation Conference, Loughborough, UK, 2007, pp. 277-280.

[22] J. Fayos-Fernandes, C. Arranz-Faz, A. MartinezGonzalez, and D. Sanchez-Hernandez, "Effect of pierced metallic objects on SAR distributions at 900MHz," Bioelectromagnetics, vol. 27, pp. 337353, 2006.

[23] A. Taflove, Computational electrodynamics. The finite-difference time-domain method: Artech House, Inc., 1995.

[24] J. P. Berenger, "A perfectly matched layer for the absorption of electromagnetic waves," Journal of Computational Physics, vol. 114, pp. 185-200, 1994.

[25] P. J. Dimbylow and O. P. Gandhi, "Finite-difference time-domain calculations of SAR in a realistic heterogeneous model of the head for plane-wave exposure from $600 \mathrm{MHz}$ to $3 \mathrm{GHz}, "$ Physics in Medicine and Biology, vol. 36, pp. 1075-1089, 1991.

[26] K. Caputa, M. Okoniewski, and M. A. Stuchly, "An algorithm for computations of the power deposition in human tissue," IEEE Antennas and Propagation Magazine, vol. 41, pp. 102-107, 1999.

[27] C. J. Panagamuwa, W. Whittow, R. Edwards, J. C. Vardaxoglou, and P. McEvoy, "A study of the validation of RF energy Specific Absorption Rates for simulations of anatomically correct head FDTD simulations and truncated DASY4 standard equipment measurements," in The First European Conference on Antennas and Propagation (EuCAP 2006), Nice, France, 2006.

[28] C. J. Panagamuwa, W. G. Whittow, R. M. Edwards, and J. C. Vardaxoglou, "Experimental verification of a modified specific anthropomorphic mannequin (SAM) head used for SAR measurements," in 2007 Loughborough Antennas and Propagation Conference, Loughborough, UK, 2007, pp. 261-264.

[29] K. S. Nikita, M. Cavagnaro, P. Bernardi, N. K. Uzunoglu, S. Pisa, E. Piuzzi, J. N. Sahalos, G. I. Krikelas, J. A. Vaul, P. S. Excell, G. Cerri, S. Chiarandini, R. De Leo, and P. Russo, "A study of uncertainties in modeling antenna performance and power absorption in the head of a cellular phone user," IEEE Transactions on Microwave Theory and Techniques, vol. 48, pp. 2676-2685, 2000.

[30] P. Bernardi, M. Cavagnaro, S. Pisa, and E. Piuzzi, "SAR distribution and temperature increase in an anatomical model of the human eye exposed to the field radiated by the user antenna in a wireless LAN," IEEE Transactions on Microwave Theory and Techniques, vol. 46, pp. 2074-2082, 1998.

C. J. Panagamuwa, W. G. Whittow, R. M. Edwards, and J. C. Vardaxoglou, "A study of the effects of metallic pins on SAR using a specific anthropomorphic mannequin (SAM) head phantom," in The Second European Conference on Antennas and Propagation (EuCAP 2007) Edinburgh, UK, 2007. 